\title{
The hybrid uncertain neural network method for mechanical reliability analysis
}

\author{
Wensheng Peng*, Jianguo Zhang** and Lingfei You*** \\ School of Reliability and Systems Engineering, Beihang University, Beijing 100191, China \\ Science and Technology on Reliability and Environment Engineering Laboratory, Beihang University, Beijing 100191, China
}

\begin{abstract}
Concerning the issue of high-dimensions, hybrid uncertainties of randomness and intervals including implicit and highly nonlinear limit state function, reliability analysis based on the hybrid uncertainty reliability mode combining with back propagation neural network (HU-BP neural network) is proposed in this paper. Random variables and interval variables are as input layer of the neural network, after the training and approximation of the neural network, the response variables are obtained through the output layer. Reliability index is calculated by solving the optimization model of the most probable point (MPP) searching in the limit state band. Two numerical cases are used to demonstrate the method proposed in this paper, and finally the method is employed to solving an engineering problem of the aerospace friction plate. For this high nonlinear, small failure probability problem with interval variables, this method could achieve a good analysis result.
\end{abstract}

Key words: neural network, interval variables, hybrid uncertainty model, aerospace mechanism, nonlinearity reliability.

\section{Introduction}

Traditional reliability analysis requires the availability of probability distributions. In many engineering applications, the distributions of some variables may not be precisely known due to limited information. These variables are known with certain intervals, in other words, the hybrid uncertain model with probabilistic and interval widely exists in the practical engineering problems. Some researches make some assumptions for random distributions when using probability approach to perform the reliability analysis[1-3], and these methods bring a problem of confidence. Other techniques[4-8] also are proposed to deal with this problem. Literature[8] analyze the hybrid reliability of linear problems through a two-stage limit state function. A robust design method combining the probability and non-probability is also developed for uncertain structures[9-11]. More studies on the precise hybrid probability and interval can refer to[12-17].

All approaches for the hybrid uncertain model above are based on the explicit performance function. However, in engineering application, most problems are implicit. It means that we could not analyze reliability on the performance function directly. Response surface method (RSM) is widely used in both probability and non-probabilistic reliability problems[18,19]. A polynomial function is used to approximate the unknown implicit performance function. This technique develops well in probabilistic reliability analysis, and fairly accurate estimate of the failure probability could be obtained if the selected polynomial function fits the actual limit state well. Some researches also discuss the application of RSM in non-probabilistic reliability analysis[20]. However, for the mechanical systems, the performance function is highly nonlinear, the polynomial based RSM is not accurate enough for the real limit state function. Some researchers [21-23] also focus on the stochastic expansion method for the mixed uncertainties, but these methods are still based on the probabilistic assumption to some extent. Artificial neural network(ANN) algorithm has been rapidly developed for universal function approximations[24-26] ANN has the stronger learning ability for fitting complex
This is an Open Access article distributed under the terms of the Creative Commons Attribution Non-Commercial License (http://creativecommons.org/licenses/by$\mathrm{nc} / 3.0 /$ which permits unrestricted non-commercial use, distribution, and reproduction in any medium, provided the original work is properly cited. (c) * Ph. D Candidate, Corresponding author: wshpeng@buaa.edu.cn
** Professor and Ph.D. *** Master Candidate 
problems. The ANN technique was compared with the RSM and other methods for structural reliability analysis[27]. Literature performed reliability analysis of a mine pillar and geotechnical engineering structure by combining finite element model (FEM)s, ANN, first order reliability method (FORM), second order reliability method(SORM) and Monte-Carlo simulation[28-30].

In this paper, a hybrid uncertainty reliability analysis methodbased on theANNisproposed. Thehybriduncertainty reliability model combines with the back propagation (HUBP) neural network for highly nonlinear performance function reliability problems. The paper is organized as follows: the probability-interval hybrid reliability theory and ANN theory are introduced in section2 and section 3 respectively; HU-BP neural network is constructed in section 4; section 5 proposes the reliability analysis based HU-BP neural network; two numerical cases and an engineering case are carried out in section 6 to demonstrate the method, and finally is conclusion in section 7 .

\section{Probability-interval hybrid reliability theory}

Assuming that $X=\left(X_{1}, X_{2}, \ldots, X_{n}\right)$ is an $n$-dimensional random vector, and $Y=\left(Y_{1}, Y_{2}, \ldots, Y_{m}\right)$ is an $m$-dimensional interval vector. The limit state function $Z$ is defined as follows:

$$
Z=g(\boldsymbol{X}, \boldsymbol{Y})=0
$$

The failure probability $P_{\mathrm{f}}$ can be defined as

$$
P_{\mathrm{f}}=\operatorname{Pr}\{g(\boldsymbol{X}, \boldsymbol{Y}) \leq 0\}
$$

Where $\operatorname{Pr}\{\bullet\}$ represents the probability. The interval parameters $Y_{i}, i=1,2, \ldots, m$ can be described as the following equations:

$$
\begin{aligned}
& Y_{i}=\left[Y_{i}^{\mathrm{L}}, Y_{i}^{\mathrm{U}}\right] \\
& Y_{i}^{\mathrm{c}}=\frac{Y_{i}^{\mathrm{L}}+Y_{i}^{\mathrm{U}}}{2}, Y_{i}^{\mathrm{r}}=\frac{Y_{i}^{\mathrm{U}}-Y_{i}^{\mathrm{L}}}{2}, \gamma_{i}=\frac{Y_{i}^{\mathrm{r}}}{Y_{i}^{\mathrm{c}}} \quad i=1,2, \cdots, m
\end{aligned}
$$

Where $Y_{i}^{L}, Y_{i}^{U}, Y_{i}^{c}, Y_{i}^{r}$ represent the lower bound, the upper bound, the middle point, the radius of the interval variables $Y_{i} \cdot \gamma_{i}$ represents the degree of uncertainty of the $Y_{i}$.

In the limit state function $Z$, the random variables as well as interval variables are included. The random parameter space will form a band of limit state when $Z=0$. In this limit state band, the boundaries can be represented as

$$
\begin{aligned}
& \max _{\boldsymbol{Y}} g(\boldsymbol{X}, \boldsymbol{Y})=0 \\
& \min _{\boldsymbol{Y}} g(\boldsymbol{X}, \boldsymbol{Y})=0
\end{aligned}
$$

$\max _{Y} g(X, Y)$ and $\min _{Y} g(X, Y)$ are the maximum and minimum values of the performance function g over the intervals of $Y$. Thus the failure probability $P_{\mathrm{f}}$ can be given as

$$
\begin{aligned}
& P_{\mathrm{f}}=\left[P_{\mathrm{f}}^{\mathrm{L}}, P_{\mathrm{f}}^{\mathrm{U}}\right] \\
& P_{\mathrm{f}}^{\mathrm{L}}=\operatorname{Pr}\left\{\max _{\boldsymbol{Y}} g(\boldsymbol{X}, \boldsymbol{Y})<0\right\} \\
& P_{\mathrm{f}}^{\mathrm{U}}=\operatorname{Pr}\left\{\min _{\boldsymbol{Y}} g(\boldsymbol{X}, \boldsymbol{Y})<0\right\}
\end{aligned}
$$

Where $P_{\mathrm{f}}^{L}$ and $P_{\mathrm{f}}^{U}$ represent the lower and upper bounds of the failure probability, respectively

The limit state band of the failure is as shown as in Fig. 1. When using classical FORM or SORM to solve the hybrid uncertainty model with both random variables and interval variables, the reliability index will belong to an interval and two optimization problems:

$$
\begin{aligned}
& \begin{cases}\beta^{\mathrm{U}}=\min _{\boldsymbol{U}}\|\boldsymbol{U}\| \\
\text { s.t. } & \max _{Y} G(\boldsymbol{U}, \boldsymbol{Y})=0\end{cases} \\
& \begin{cases}\beta^{\mathrm{L}}= & \min _{U}\|\boldsymbol{U}\| \\
\text { s.t. } & \min _{\boldsymbol{Y}} G(\boldsymbol{U}, \boldsymbol{Y})=0\end{cases}
\end{aligned}
$$

Where $\beta^{\mathrm{U}}$ and $\beta^{\mathrm{L}}$ represent the upper and lower bounds of the reliability index, respectively. $U$ is the normalization of $X$, and the normalization process is as :

$$
\begin{aligned}
& \Phi\left(U_{i}\right)=F_{X_{i}}\left(X_{i}\right), i=1,2, \cdots, n \\
& U_{i}=\Phi^{-1}\left[F_{X_{i}}\left(X_{i}\right)\right], i=1,2, \cdots, n
\end{aligned}
$$

\section{Artificial Neural network technique}

The multi-layer perceptron, trained by the back propagation(BP), is currently the most widely used ANN. In this context, ANN is referred to as multi-layer perceptron or multi-layer feed forward ANN. An ANN consists of a set of neurons that are logically arranged into the input layer, the output layer and the hidden layer. The neurons is a processing elements whose output is calculated by multiplying its inputs by a weight vector, summing the results, and applying

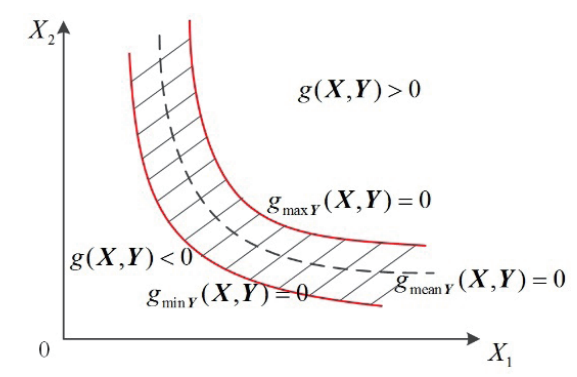

Fig. 1. The limit state band of the hybrid model 
an activation function to the sum.

$$
y=f\left[\sum_{k=1}^{n} x_{k} w_{k}+b_{k}\right]
$$

Where $w_{k}$ is the weight coefficient, $b_{k}$ is threshold. The active function can be linear or non-linear. A linear activation function's output is simply equal to its input:

$$
f(x)=x
$$

The most common non-linear activation function is the logistic sigmoidal function

$$
f(x)=\frac{1}{1+\exp (-\delta x)}
$$

Where $\delta$ is called the sigmoid slope, usually $\delta=1$

\section{Hybrid uncertainty based BP neural network}

In this section, a hybrid uncertainty based $\mathrm{BP}(\mathrm{HU}-\mathrm{BP})$ neural network is defined. In order to illustrate the HU-BP neural network, this paper will use the typical three-layer neural network. Its structure is as shown in Fig. 2. There are input layer, hidden layer and output layer in the neural network. As mentioned in the section 2, in reliability analysis, the hybrid uncertainties of probability and interval are widely exist in the engineering applications, and the performance function as equation (1), there are random variables $X=\left(X_{1}\right.$, $\left.X_{2}, \ldots, X_{n}\right)$ and $Y=\left(Y_{1}, Y_{2}, \ldots, Y_{m}\right)$ in the performance function $g$.

Then the performance function of the HU-BP neural network could be defined as follows:

$$
g(\boldsymbol{X}, \boldsymbol{Y})=f_{2}\left[\boldsymbol{W}_{2}^{\mathrm{T}} f_{1}\left(\boldsymbol{W}_{1}^{\mathrm{T}}(\boldsymbol{X}, \boldsymbol{Y})\right)\right]
$$

Where $W_{1}$ and $W_{2}$ represent the weight coefficients of the transformation.

In above equations, the "training rules" of the neural network is defined, and the next issue is the training process. In the training process, training samples for neural network is important.

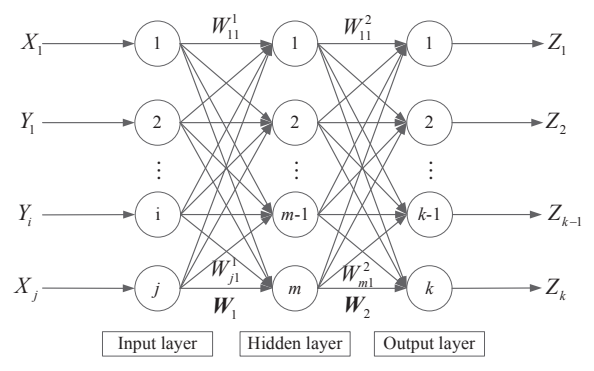

Fig. 2. A three-layer HU-BP neural network
This paper uses the improved axial experimental design method[31] to obtain samples points of $X$ and $Y$, the initial sample point is selected as $\left(\mu_{X}, Y^{C}\right)$, where $\mu_{X}$ is the mean vector of the random variables $X$ and $Y^{C}$ is the midpoint vector of the interval variables $Y$, the samples of input variables $(\overline{\boldsymbol{X}}, \overline{\boldsymbol{Y}})$ will be updated in subsequent iterations, the remaining $2 n+2 m$ samples points will be obtained through $\bar{X}_{i} \pm \kappa_{x} \cdot \sigma_{X_{i}}, i=1,2, \ldots, n$ and $\bar{Y}_{j} \pm \kappa_{y} \cdot Y_{j}^{\mathrm{r}}, j=1,2, \ldots, m$, where $\sigma_{X_{i}}$ is the standard deviation of $X_{i}$ and $\mathrm{Y}_{j}^{\mathrm{r}}$ is the radius of $\mathrm{Y}_{j}, \kappa_{x}$ and $\kappa_{y}$ are two sampling coefficients and generally between $[1,3]$ can provide good computational results.

Once the input samples are identified , the output samples will obtained by FEA of the mechanical systems. And finally the train samples $\left(\left(X^{(t)}, Y^{(t)}\right), Z^{(t)}\right)$ are obtained.

The whole training steps of the HU-BP neural network are: Step 1: built the structure of the BP neural network.

Step 2: select the hybrid uncertainty training sample sets $\left(\left(X^{(t)}, Y^{(t)}\right), Z^{(t)}\right),(t=1,2, \ldots, S)$ according to section 4 , and identify the initial weight coefficient $W_{k}^{(0)}$

Step 3: calculate $Z^{(t)}$ with the trained $\operatorname{set}\left(X^{(t)}, Y^{(t)}\right)$

Step 4: reverse calculate the partial derivative $\frac{\partial e^{(t)}}{\partial \boldsymbol{W}_{k}^{(t)}}$, $(k=1,2, \ldots, L)$, where $e$ is the final error, and $e=\frac{1}{2}\left\|\boldsymbol{Z}_{L}-\boldsymbol{T}\right\|=\frac{1}{2}\left(\boldsymbol{Z}_{L}-\boldsymbol{T}\right)^{\mathrm{T}}\left(\boldsymbol{Z}_{L}-\boldsymbol{T}\right), T$ is the ideal output of the neural network.

Step 5: the modified $W_{k}^{(t+1)}$ is obtained by $\boldsymbol{W}_{k}^{(t+1)}=\boldsymbol{W}_{k}^{(t)}+\delta \boldsymbol{W}_{k}^{(t)}, \delta \boldsymbol{W}_{k}^{(t)}=-\eta_{t} \frac{\partial e^{(t)}}{\partial \boldsymbol{W}_{k}^{(t)}}, \mathrm{k}=1,2, \ldots, \mathrm{L}, \eta_{t}$ is the training efficient in $k^{\text {th }}$ step.

Step 6: repeat the step 3 to 5 until all the samples are trained.

\section{Reliability analysis based HU-BP neural network}

The implicit limit state function containing hybrid uncertainty of probability and interval can be simultaneously

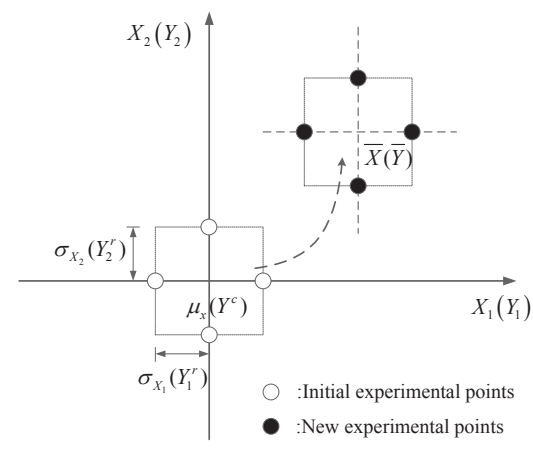

Fig. 3. Modified axial experimental design method for $\mathrm{HU}$ 
approximated through HU-BP neural network, then the method of HU-BP neural network based on "Most Probable Point" (MPP) is used to calculated the failure probability.

The limit state band of the failure is as shown as in Fig. 4. When using classical FORM or SORM to solve the hybrid uncertainty model with both random variables and interval variables, the reliability index will belong to an interval and two optimization problems:

$$
\begin{aligned}
& \begin{cases}\beta^{U}= & \min _{U}\|\boldsymbol{U}\| \\
\text { s.t. } & \max _{Y} G(\boldsymbol{U}, \boldsymbol{Y})=0\end{cases} \\
& \begin{cases}\beta^{\mathrm{L}}= & \min _{\boldsymbol{U}}\|\boldsymbol{U}\| \\
\text { s.t. } & \min _{\boldsymbol{Y}} G(\boldsymbol{U}, \boldsymbol{Y})=0\end{cases}
\end{aligned}
$$

For the MPP search in the equation (14), HL-RF algorithm is widely used, and reference[32] has proposed an iHLRF algorithm for limit state function containing random variables and interval variables. For example, we focus on the MPP for $\beta^{\mathrm{U}}$, in iteration $k+1$, the MPP is given by

$$
\boldsymbol{U}_{k+1}=\boldsymbol{U}_{k}+\alpha \boldsymbol{d}_{k}
$$

Where the search direction $d_{k}$ is defined by

$$
\boldsymbol{d}_{k}=\frac{\nabla g_{Y}\left(\boldsymbol{U}_{k}, \boldsymbol{Y}_{k}\right) \boldsymbol{U}_{k}^{\mathrm{T}}-g\left(\boldsymbol{U}_{k}, \boldsymbol{Y}_{k}\right)}{\left\|\nabla g_{Y}\left(\boldsymbol{U}_{k}, \boldsymbol{Y}_{k}\right)\right\|^{2}} \nabla g_{Y}\left(\boldsymbol{U}_{k}, \boldsymbol{Y}_{k}\right)-\boldsymbol{U}_{k}
$$

Where

$$
\nabla g_{Y}\left(\boldsymbol{U}_{k}, \boldsymbol{y}_{k}\right)=\left(\frac{\partial g}{\partial U_{1}}, \frac{\partial g}{\partial U_{2}}, \cdots, \frac{\partial g}{\partial U_{n}}\right)_{U_{k}, Y_{k}}
$$

The step size $\alpha$ is determined by minimizing the merit function defined by

$$
m(\boldsymbol{U}, \boldsymbol{Y})=\frac{1}{2}\|\boldsymbol{U}\|+c|g(\boldsymbol{U}, \boldsymbol{Y})|
$$

In which the constant $\mathrm{c}$ should satisfy

$$
c>\frac{\|\boldsymbol{U}\|}{\|\nabla g(\boldsymbol{U}, \boldsymbol{Y})\|}
$$

In above equations, intervals are assumed fixed, once the

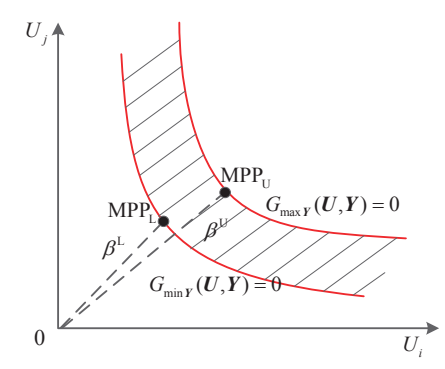

Fig. 4. Reliability index of the HU reliability model
$U_{k+1}$ is found, the interval analysis is conducted to find $Y_{k+1}$ that maximize the performance function. The optimization model is given by

$$
\max _{Y} g\left(\boldsymbol{U}_{k+1}, \boldsymbol{Y}\right) \mid \boldsymbol{Y} \in C
$$

where $C$ represents the constrained interval real field. Then, the reliability analysis steps for HU-BP neural network are as follows:

Step 1: suppose that $X=\left(X_{1}, X_{2}, \ldots, X_{n}\right)$ is the basic $\mathrm{n}$-dimensional random variables in original coordinate system, then vector $U=\left(U_{1}, U_{2}, \ldots, U_{n}\right)$ represents the random variables which are equivalent standard normal space. $Y=\left(Y_{1}, Y_{2}, \ldots, Y_{m}\right)$ is $m$-dimensional interval variables.

Step 2: construct an approximate BP neural network for the structure reliability, and select train sample sets

Step 3: establish the MPP optimization model of the performance function $G(U, Y)$ and calculate the partial derivatives of design point using HU-BN neural network $\left.\frac{\partial g}{\partial X}\right|_{x_{i}^{*}}$, and then the optimization model of equation (19) is obtained;

Step 4: find $Y_{k+1}$ by solving the optimization model of equation (19);

Step 5: check convergence, if $\left|g\left(\boldsymbol{U}_{k+1}, \boldsymbol{Y}_{k+1}\right)\right| \leq \varepsilon_{1}$ and $\left\|\boldsymbol{U}_{k+1}-\boldsymbol{U}_{k}\right\| \leq \varepsilon_{2}\left(\varepsilon_{1}\right.$ and $\varepsilon_{2}$ are small positive numbers $\left.=1.0\right)$, then $\beta^{\text {min }}=\left\|\boldsymbol{U}_{k+1}\right\|$ and go to step 6 , otherwise, $k=k+1$, go to step 3

Step 6: $P_{f}^{\min }=\Phi\left(-\beta^{\min }\right)$

\section{Case study}

\section{1 numerical case 1}

Denote the reliability function as $\mathrm{g}$, and $\mathrm{g}$ is given by

$$
g=\frac{x_{1} \cdot x_{2}^{2} \cdot y_{1}}{\left(\sqrt{y_{2}}-1\right)\left(y_{1}-x_{1}\right)}+\exp \left[0.1\left(x_{1}+x_{2}-1\right)+1\right]-\exp \left[0.18\left(y_{1}-y_{2}\right)+2.5\right]+14
$$

Where the above explicit limit state function is assumed to be an implicit model for simulation. And the uncertainty information of variables of $x_{1}, x_{2}, y_{1}, y_{2}$ are : $x_{1}$ and $x_{2}$ are random variables, they obey normal distribution, their mean value standard deviation are: $\mu_{x_{1}}=1.2, \sigma_{x_{1}}=0.12 ; \mu_{x_{1}}=0.8$, $\sigma_{x_{1}}=0.02 . y_{1}$ and $y_{2}$ are interval variables, and $y_{1} \in[4,4.5]$, $y_{2} \in[1.6,1.64]$.

The HU-BP neural network is employed to fitting the performance, and 120 samples $\left(x_{i}, y_{i}\right)$ are used, and equation (20) is used to compute the response value $z_{i}=g\left(x_{i}, y_{i}\right)$, the train sample sets $\left\{\left(x_{i}, y_{i}\right), z_{i}\right\}$ are obtained. A three-layer HUBP neural network is employed and the transfer function in hidden layer is chosen as 


$$
f_{\text {tan sig }}(x)=\tanh x
$$

The output layer function is :

$$
f_{\text {purlin }}(x)=x
$$

Then the fitting results of HU-BP neural network is as shown in Fig. 5 and Fig. 6.

The reliability index of the performance with HU-BP neural network is $\beta^{\mathrm{L}}=3.30744 ; \beta^{\mathrm{U}}=3.59621$. In order to illustrate the method proposed by this paper furthermore, the second order RSM is also employed in the same problem. In the RSM, $y_{1}$ and $y_{2}$ are sampled as the unified in the intervals and the two MPP is also calculated. After 8 iterations, the reliability index are $\beta^{\mathrm{L}}=3.85176 ; \beta^{\mathrm{U}}=4.158613$. Comparing the results, it can be seen that the reliability indexes obtained by the HU-neural network are small than the results obtained by the RSM. It demonstrates that the performance function obtained by the neural network is more accurate than the RSM. In order to verify the results, the failure probability of this problem is calculated by the direct MC with 1000000 samples, and the max probability and min probability are

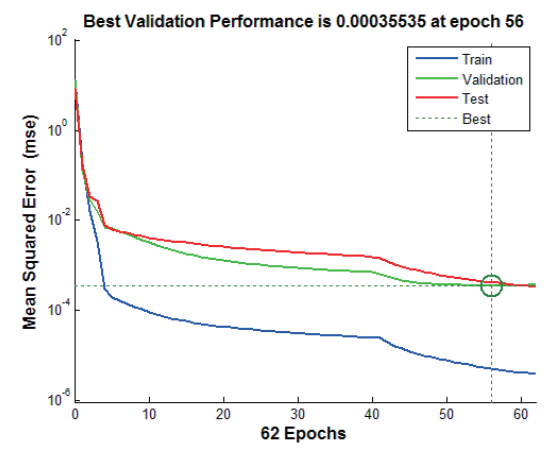

Fig. 5. Fitting result of HU-BP neural network
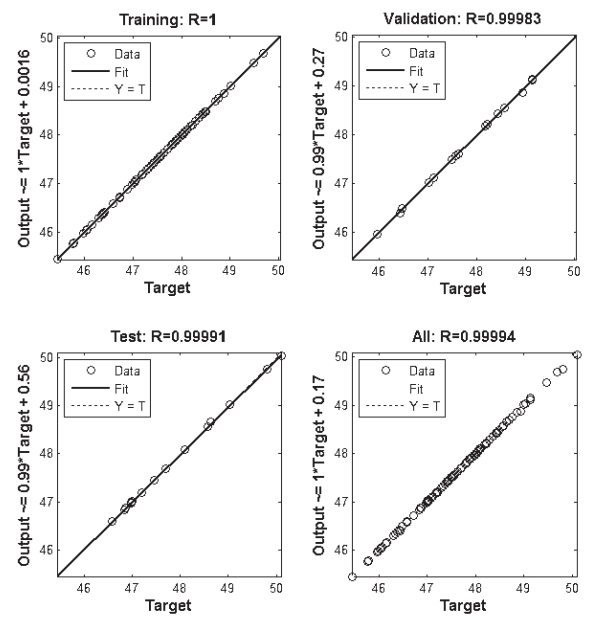

Fig. 6. Fitting results of performance function as list in table 1. It can be seen that the failure probability of $P_{\mathrm{f}}^{\mathrm{U}}$ and $P_{\mathrm{f}}^{\mathrm{L}}$ obtained through the HU-BP neural network and the MC is almost the same, the relative error of reliability are: $0.132 \mathrm{e}-4$ and $0.455 \mathrm{e}-4$. The relative error of results of the RSM is larger, and they are $4.369 \mathrm{e}-4$ and $2.524 \mathrm{e}-4$ respectively. The results indicate that the HU-BP neural could get a good result in the reliability analysis

\section{2 numerical case 2}

The performance function of ten-bar truss structure as shown in Fig. 7 is

$$
Z=4-u_{2 y}\left(A, F_{1}, F_{2}, F_{3}\right)=0
$$

Where $\mathrm{u}_{2 y}$ is the horizontal displacement $(/ \mathrm{m})$ of the joint 2 . The length of the horizontal and vertical bars is 1.0 m. Joint 4 is subjected to the vertical load $F_{1}$ and Joint 2 is subjected to the horizontal load $F_{3}$ and vertical load $F_{2}$. The Young's Modulus of the material $E$ is normal variable, $\mu_{E}=2.0 \times 106 \mathrm{kN} / \mathrm{m}^{2}$, variation coefficient $V_{E}=0.01$. The mean value and variation coefficient are $\mu_{A_{i}}=0.36 \mathrm{~m}^{2}, V_{A_{i}}=0.1$. The external load $F_{3}$ obeys normal distribution, the mean value and variation coefficient are $: \mu_{F_{3}}=10 \mathrm{~N}, V_{F_{3}}=0.1$. The external loads $F_{1}, F_{2}$, are interval uncertain, and $F_{1} \in[76,84] \mathrm{kN}$, $F_{2} \in[9.5,10.5]$. Now we calculate the failure probability of the structure.

For this problem, the displacement $u_{2 y}$ could be obtained through the FEA, and the HU-BP neural network method proposed by this paper is utilized for reliability analysis. To fitting the performance function, 150 samples $\left(x_{i}, y_{i}\right)=\left(A, F_{1}\right.$, $\left.F_{2}, F_{3}\right)^{\mathrm{T}}$ are used as input variables, and FEA model is used to compute the response value $z_{i}=4-u_{2 y}\left(A, F_{1}, F_{2}, F_{3}\right)$. The

Table 1. Failure probability of different methods

\begin{tabular}{lll}
\hline Methods & $P_{\mathrm{f}}^{\mathrm{U}}$ & $P_{\mathrm{f}}^{\mathrm{L}}$ \\
\hline Method proposed in this paper & $4.834 \mathrm{e}-4$ & $2.326 \mathrm{e}-4$ \\
\hline RSM method & $5.91 \mathrm{e}-5$ & $2.57 \mathrm{e}-5$ \\
\hline MC & $4.966 \mathrm{e}-4$ & $2.781 \mathrm{e}-4$ \\
\hline
\end{tabular}

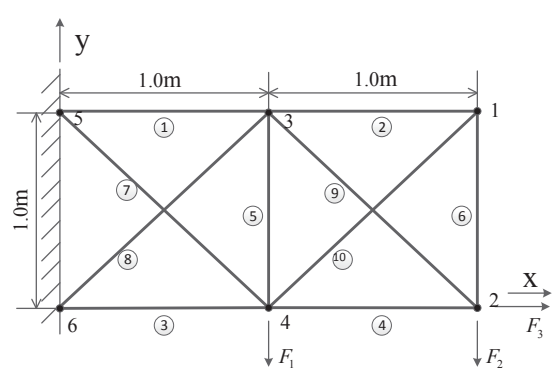

Fig. 7. Ten-bar plane structure 
train sample sets $\left\{\left(x_{i}, y_{i}\right), z_{i}\right\}$ are obtained in a three-layer HU-BP neural network. After 12 iterations, the reliability index of this problem are obtained as: $\beta^{\mathrm{U}}=3.4721 ; \beta^{\mathrm{L}}=3.3122$. For the same problem, second order RSM and Monte Carlo simulation are also utilized here to compute the reliability, the RSM with 150 sample sets for fitting the performance and the results of failure probability are listed in table 2 . The RSM method has 8 iterations for reliability index but it is rough than the results obtained by the method proposed by this paper. The whole computational time of the RSM is 0.79 hours, and the whole computational time of the HU-BP neural network is 1.71 hours (the computer with Intel CORE 5 CPU and 3.00GHz). The MC based on ANN method with 100000 sample sets, and the computational time is 16 hours. The results indicate that the HU-neural network could get a more precise result for high nonlinearity problem, but it cost more time than the RSM method. However, it is much more efficient than the MC method. It means that the HU-BP neural network is meaningful in the implicit high nonlinearity problems

Table 2. Reliability of different methods

\begin{tabular}{lll}
\hline Methods & $R^{\mathrm{U}}$ & $R^{\mathrm{L}}$ \\
\hline Method proposed in this paper & 0.9997398 & 0.9995335 \\
\hline RSM & 0.9998282 & 0.9996631 \\
\hline MC based ANN & 0.9997279 & 0.9995754 \\
\hline
\end{tabular}

\section{3 engineering case}

The reliability analysis of locking mechanism in aerospace engineering.

The locking mechanism as shown in Fig. 8, it is a key part in support device which is used as the main power transmission structure between the spacecraft and transparency nose. The locking mechanism works by contacting the inner friction plates(IFP) and the outer friction plates(OFP). The friction force between IFP and OFP is achieved by the shear stress of the IFP underlying the load and the contact area $S$. Now we analyze the friction force reliability of the locking mechanism. Referring relative tests and discussions, the variables of the performance function are as shown in table 3

The locking mechanism is pressured with the normal load $P_{1}$ and the lateral load $P_{2}$, and their values are as shown in Fig. 9. Obviously this is an implicit high nonlinear problem, the HU-BP neural network method and FEA is combined to solve this problem. Finite element model of the locking

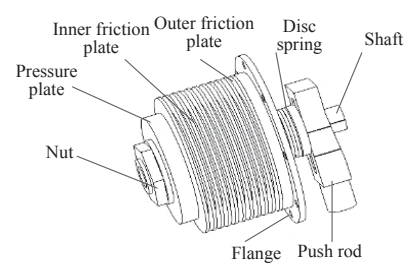

Fig. 8. Structure of the locking mechanism

Table 3. Thecharacteristic parameters of the locking mechanism

\begin{tabular}{lll}
\hline Parameters & Physical meaning & Values \\
\hline$E_{1}(\mathrm{Gpa})$ & elastic modulus of IFP & 65.4 \\
\hline $\mathrm{r}$ & Passion rate & 0.3 \\
\hline$\rho\left(\mathrm{kg} / \mathrm{m}^{3}\right)$ & density & $2.72 \mathrm{e} 3$ \\
\hline$G_{1}(\mathrm{GPa})$ & Shear modulus of IFP & 25.15 \\
\hline$E_{2}(\mathrm{Gpa})$ & elastic modulus of OFP & 70 \\
\hline$G_{2}(\mathrm{GPa})$ & Shear modulus of OFP & 24.81 \\
\hline$c_{1}$ & Static friction coefficient & 0.59 \\
\hline$c_{2}$ & viscous damping coefficient & $0.15878 \mathrm{e} 9$ \\
\hline
\end{tabular}
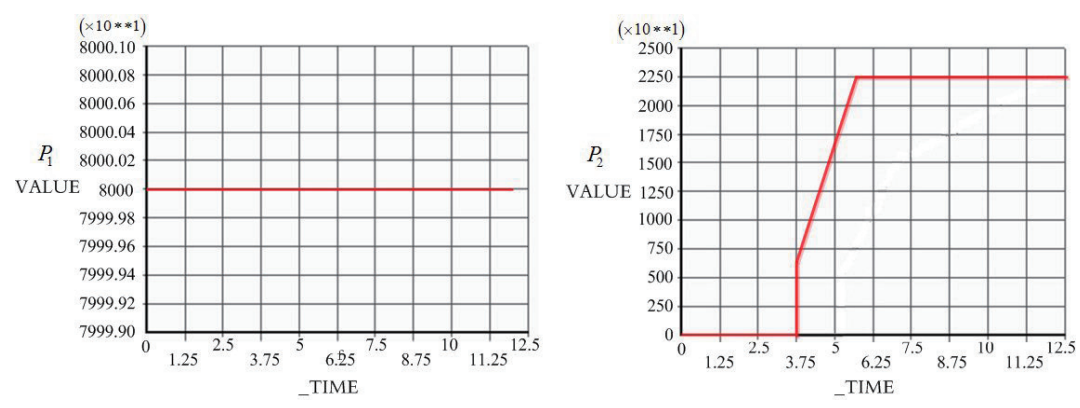

Fig. 9. Values of $P_{1}$ and $P_{2}$ 
mechanism can be established with ANSYS, and the work simulation process of contact and shock is carried out in the LS-DYNA. In the loading process, the $P_{1}$ is loading on the surface of the flange plate, and the $P_{2}$ is loading on side face

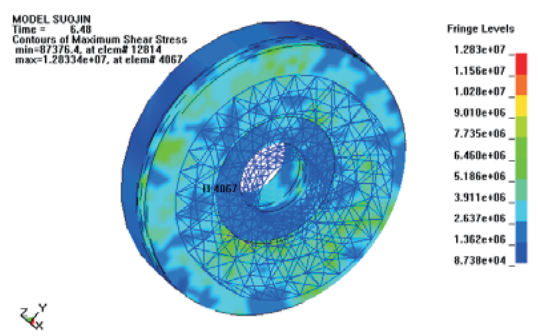

Fig. 10. Shear stress of the friction plate

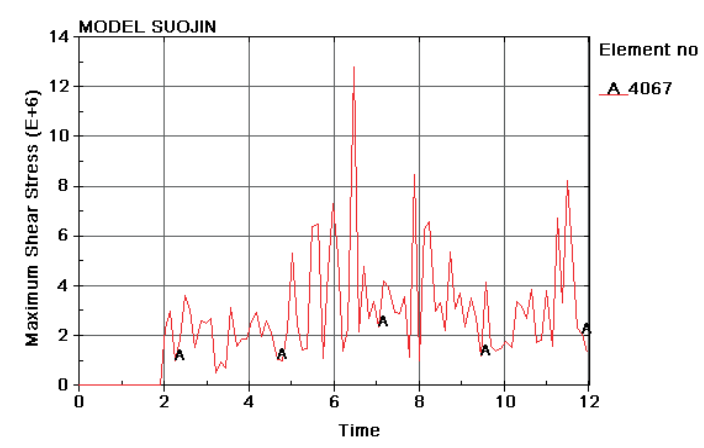

Fig. 11. Curve of shear stress with time of the OFP. The boundary condition is that the all the freedom degrees of the Pressure plate are constrained. In Fig. 10, the maximum shear stress is $12.83 \mathrm{MPa}$, and the stress varying by the time is as shown in Fig. 11. Through the discretization of time interval into 84 time point, the average shear stress could be obtained as $3.1 \mathrm{Mpa}$ according to the table 4 .

-Reliability simulation analysis for locking mechanism

The definition of limit state function and probabilistic characteristic and interval range of variables. The model of stress and strength interference and hybrid uncertainty performance function is applied to compute the failure probability. The limit state function is defined as

$$
Z=F-F_{0}=f\left(P_{1}, P_{2}, E_{1}, E_{2}, G_{1}, G_{2}, D\right) \times S-F_{0}
$$

Where $f\left(P_{1}, P_{2}, E_{1}, E_{2}, G_{1}, G_{2}, D\right)$ is the element average equivalent stress of the inner friction plate, which could be calculated by LS-DYNA. $S$ is the area of the plate. $F_{0}$ is the allowable load.

Based on the limit historic data and the epistemic knowledge, the probabilistic and interval characters of the variables are in table 5 .

-Reliability simulation analysis

The limit state function could be approximated by threelayer HU-BP neural network. The number of neurons for input layer is nine and there are eighteen hidden layers,

Table 4. Shear stress values with time under the varying loads

\begin{tabular}{|c|c|c|c|c|c|c|c|}
\hline No. & Value(Pa) & No. & Value(Pa) & No. & Value(Pa) & No. & Value(Pa) \\
\hline 1 & $2.30337 \mathrm{e} 6$ & 22 & $2.11395 \mathrm{e} 6$ & 43 & $2.34749 \mathrm{e} 6$ & 64 & $4.15947 \mathrm{e} 6$ \\
\hline 2 & $2.99647 \mathrm{e} 6$ & 23 & $1.05623 \mathrm{e} 6$ & 44 & $4.2094 \mathrm{e} 6$ & 65 & $1.56383 \mathrm{e} 6$ \\
\hline 3 & 997698 & 24 & $1.01825 \mathrm{e} 6$ & 45 & $3.95451 \mathrm{e} 6$ & 66 & $1.41306 \mathrm{e} 6$ \\
\hline 4 & $1.86108 \mathrm{e} 6$ & 25 & $2.2757 \mathrm{e} 6$ & 46 & $2.96955 \mathrm{e} 6$ & 67 & $1.47164 \mathrm{e} 6$ \\
\hline 5 & $3.61296 \mathrm{e} 6$ & 26 & $5.35305 \mathrm{e} 6$ & 47 & $2.90995 \mathrm{e} 6$ & 68 & $1.79964 \mathrm{e} 6$ \\
\hline 6 & $3.03982 \mathrm{e} 6$ & 27 & $2.44242 \mathrm{e} 6$ & 48 & $3.59123 \mathrm{e} 6$ & 69 & $1.532 \mathrm{e} 6$ \\
\hline 7 & $1.53638 \mathrm{e} 6$ & 28 & $1.45343 \mathrm{e} 6$ & 49 & $1.13604 \mathrm{e} 6$ & 70 & $3.38367 \mathrm{e} 6$ \\
\hline 8 & $2.59281 \mathrm{e} 6$ & 29 & $1.51117 \mathrm{e} 6$ & 50 & $8.51143 \mathrm{e} 6$ & 71 & $3.20651 \mathrm{e} 6$ \\
\hline 9 & $2.50227 \mathrm{e} 6$ & 30 & $6.42199 \mathrm{e} 6$ & 51 & 976126 & 72 & $2.68522 \mathrm{e} 6$ \\
\hline 10 & $2.711 \mathrm{e} 6$ & 31 & $6.51172 \mathrm{e} 6$ & 52 & $6.31719 \mathrm{e} 6$ & 73 & $3.87357 \mathrm{e} 6$ \\
\hline 11 & 508333 & 32 & $1.0959 \mathrm{e} 6$ & 53 & $6.61351 \mathrm{e} 6$ & 74 & $1.71388 \mathrm{e} 6$ \\
\hline 12 & 952073 & 33 & $4.63751 \mathrm{e} 6$ & 54 & $2.98379 \mathrm{e} 6$ & 75 & $1.80908 \mathrm{e} 6$ \\
\hline 13 & 709136 & 34 & $7.31972 \mathrm{e} 6$ & 55 & $3.35098 \mathrm{e} 6$ & 76 & $3.83426 \mathrm{e} 6$ \\
\hline 14 & $3.16505 \mathrm{e} 6$ & 35 & $5.2669 \mathrm{e} 6$ & 56 & $2.2195 \mathrm{e} 6$ & 77 & $1.58045 \mathrm{e} 6$ \\
\hline 15 & $1.56832 \mathrm{e} 6$ & 36 & $1.38007 \mathrm{e} 6$ & 57 & $5.40534 \mathrm{e} 6$ & 78 & $6.76475 \mathrm{e} 6$ \\
\hline 16 & $1.87172 \mathrm{e} 6$ & 37 & $2.30834 \mathrm{e} 6$ & 58 & $3.11468 \mathrm{e} 6$ & 79 & $3.34421 \mathrm{e} 6$ \\
\hline 17 & $1.85895 \mathrm{e} 6$ & 38 & $1.28334 \mathrm{e} 7$ & 59 & $3.75113 \mathrm{e} 6$ & 80 & $8.28679 \mathrm{e} 6$ \\
\hline 18 & $2.62145 \mathrm{e} 6$ & 39 & $2.1724 \mathrm{e} 6$ & 60 & $2.35026 \mathrm{e} 6$ & 81 & $5.33554 \mathrm{e} 6$ \\
\hline 19 & $2.92965 \mathrm{e} 6$ & 40 & $4.78592 \mathrm{e} 6$ & 61 & $3.54081 \mathrm{e} 6$ & 82 & $2.3122 \mathrm{e} 6$ \\
\hline 20 & $1.95469 \mathrm{e} 6$ & 41 & $2.69129 \mathrm{e} 6$ & 62 & $2.70612 \mathrm{e} 6$ & 83 & $2.09523 \mathrm{e} 6$ \\
\hline 21 & $2.60807 \mathrm{e} 6$ & 42 & $3.41076 \mathrm{e} 6$ & 63 & $1.17879 \mathrm{e} 6$ & 84 & $1.32194 \mathrm{e} 6$ \\
\hline
\end{tabular}


Table 5. Probabilistic and interval character of variables

\begin{tabular}{llllll}
\hline Sequence number & Variables & Unit & Mean & $\begin{array}{l}\text { Coefficient } \\
\text { of variation }(\gamma)\end{array}$ & Distribution \\
\hline 1 & $P_{1}$ & $\mathrm{kN}$ & 80 & 0.1 & normal \\
\hline 2 & $P_{2}$ & $\mathrm{kN}$ & 22.5 & 0.1 & normal \\
\hline 3 & $E_{1}$ & $\mathrm{GPa}$ & 65.4 & 0.01 & normal \\
\hline 4 & $E_{2}$ & $\mathrm{GPa}$ & 70 & 0.01 & normal \\
\hline 5 & $G_{1}$ & $\mathrm{GPa}$ & 25.12 & 0.02 & normal \\
\hline 6 & $G_{2}$ & $\mathrm{GPa}$ & 24.81 & 0.02 & normal \\
\hline 7 & $\rho$ & $\mathrm{kg} / \mathrm{m}^{3}$ & $2.72 \mathrm{e} 3$ & 0.01 & normal \\
\hline 8 & $S$ & $\mathrm{~m}^{2}$ & $12.16 \mathrm{e}-3$ & 0.02 & interval \\
\hline 9 & $F_{0}$ & $\mathrm{kN}$ & 44.6 & 0.06 & interval \\
\hline
\end{tabular}

one output layer. The number of train set is 50 . The relation between root mean square error(RMSE) of neural network output vector and the number of training epochs is shown as in Fig 12 and Fig 13 .

Figure 12 shows that the lowest RMSE of the output vector in the training set can be reached after 10 epochs. After approximation, the reliability index obtained by the FORM is: $\beta_{\mathrm{Z}}^{\mathrm{L}}=3.901, \beta_{\mathrm{Z}}^{\mathrm{U}}=3.901$. The failure probability is : $\mathrm{P}_{\mathrm{f}}^{\mathrm{L}}=4.321 \mathrm{e}-6$, $\mathrm{P}_{\mathrm{f}}^{\mathrm{U}}=\mathrm{e}-6$.

To study the impact of the interval uncertainty on the failure probability, $\gamma_{s}$ and $\gamma_{F_{0}}$ are varied gradually from 0 to 0.1 and 0 to 0.15 respectively. Fig. 14 and Fig. 15 show the variation in the reliability index $\beta$ and the failure probability $P_{\mathrm{f}}$ with the change in $\gamma_{s}$ and $\gamma_{F_{0}}$. In Fig. 14, when $\gamma_{F_{0}}=0.06$ is selected, and the $\gamma_{s}$ changes from 0 to 0.1 , the reliability index changes from [4.025, 4.4076 ] to [3.719, 4.612], the

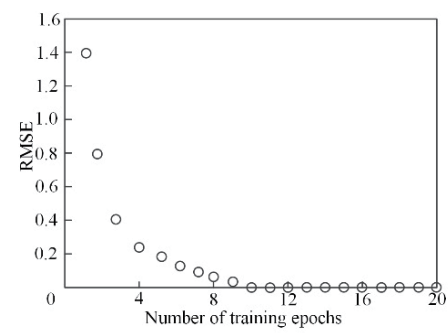

Fig. 12. RMSE of training set and the number of training epochs

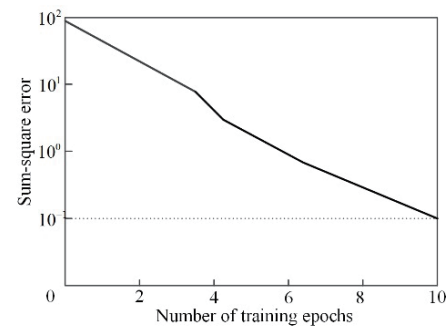

Fig. 13. Training errors vs number of epochs failure probability changes from $[5.42 \mathrm{e}-6,2.67 \mathrm{e}-5]$ to [2.02e-6, 1.042e-4 ]. In Fig. 15, when $\gamma_{s}=0.02$ is selected, and the $\gamma_{F_{0}}$ changes from 0 to 0.15 , the reliability index changes from $[4.117,4.376]$ to $[3.341,5.012]$, the failure probability changes from $[6.21 \mathrm{e}-6,1.98 \mathrm{e}-5]$ to $[3.01 \mathrm{e}-7,4.189 \mathrm{e}-4]$. This information suggests that the increase in uncertainty of the interval variables has led to an increased uncertainty in the reliability results of the whole system. And the results also show that in comparison with the $\gamma_{s}$, the impact of the uncertainty of the $\gamma_{F_{0}}$ is more significant.

\section{Conclusions}

The BP neural network for hybrid uncertainty of random variables and interval variables is constructed, and the
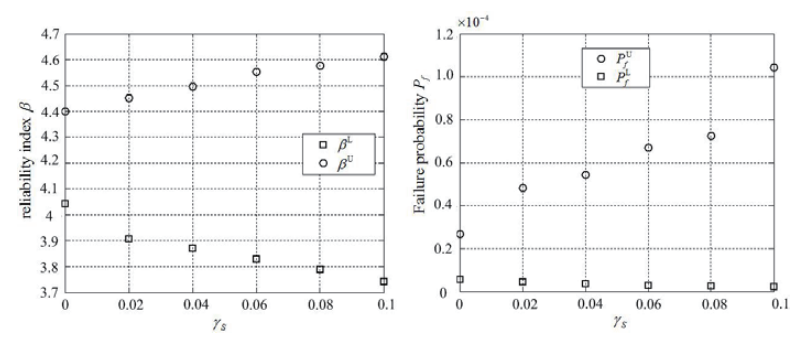

Fig. 14. effects of the uncertainty on the failure probability
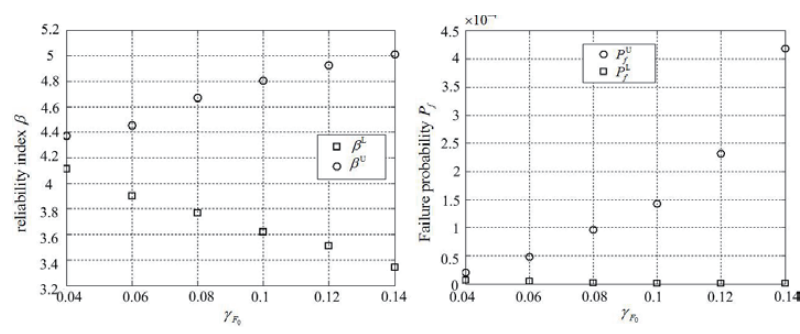

Fig. 15. Effects of the uncertainty on the failure probability 
training rules is defined in the HU-BP neural network, it is suitable for approximating high nonlinear performance function with hybrid variables;

Hybrid reliability model based analysis method combining the neural network, the limit state band is introduced concerning on the interval variables in the mechanical systems, the reliability index interval based on searching for the MPP in performance function is employed to evaluate the reliability of mechanical systems. It ignored the assumption of probability distributions of the nonprobabilistic variables and the reliability analysis is more confident in real engineering. The reliability interval has been proposed for the explicit function reliability problem, to the best of authors knowledge, this is first contribution in the implicit problems.

For high nonlinear, implicit problems of mechanical systems, the HU-BP neural network method provides an efficient and accurate way for reliability analysis. The impact of the interval uncertainty on the failure probability could also be obtained, and it is helpful for the mechanism design.

\section{Acknowledgement}

This study was supported by the National Basic Research Program of China (No. 2013CB733002).

\section{References}

[1] Hurtado, J. E. and Alvarez, D. A., "The encounter of interval and probabilistic approaches to structural reliability at design point", Applied Mechanics and Engineering, Vol. 225, 2012, pp. 74-94.

[2] Hurtado, J. E., "Assessment of reliability intervals under input distributions with uncertain parameters", Prababilistic Engineering Mechanics, Vol. 32, 2013, pp. 80-92.

[3] Ni,Z. and Qiu,Z.P. "Hybrid probabilistic fuzzy and nonprobabilistic model of structural reliability", Computers \& Industrial Engineering, Vol. 58, 2010, pp. 463-467.

[4] Jiang ,C.,Long, X. Y.,Han,X. et al, "Probability-interval hybrid reliability analysis for cracked structures existing epistemic uncertainty", Engineering Fracture Mechanics, Vol. 112-113, 2013, pp.148-164.

[5] Wang, J. and Qiu, Z. P., “The reliability analysis of probabilistic and interval hybrid structural system", Applied Mathematical Modelling, Vol. 34, 2010, pp. 3648-3658.

[6] Zhu, L. P. and Elishakoff, I., "Hybrid probabilistic and convex modeling of excitation and response of periodic structures", Mathematical Problems in Engineering, Vol. 2, No
2, pp. 143-163, 1996.

[7] Qiu, Z. P., Yang, D. and Elishakoff, I., "Probabilistic interval reliability of structural systems", International Journal of Solids and Structures, Vol. 45, No. 10, 2008, pp. 2850-2860.

[8] Guo, S. X. and Lu, Z. Z., "Hybrid probabilistic and nonprobabilistic model of structural reliability", Chinese J Mech Strength, Vol. 24, No. 4, 2002, pp. 524-526.

[9] Cheng, Y. S., Zhong, Y. X. and Zeng, G. W., "Structural robust design based on hybrid probabilistic and nonprobabilistic models", Chinese Journal of Computational Mechanics, Vol. 22, No. 4, 2005, pp. 501-505.

[10] Kang, Z. and Luo YJ., "Reliability-based structural optimization with probability and convex set hybrid models", Struct Multidisc Optim, Vol. 42, 2010, pp. 89-102.

[11] Luo, YJ., Kang, Z. and Li, A., "Structural reliability assessment based on probability and convex set mixed model". Comput Struct, Vol. 87, 2009, pp. 1408-15.

[12] Jiang, C., Li, W.X. and Han,X., et al., "Structural reliability analysis based on random distributions with interval parameters", Computer and Structures, Vol. 89, 2011, pp. 2292-2302.

[13] Du, X. P., Sudjianto, A. and Huang, B. Q., "Reliabilitybased design with the mixture of random and interval variables", inASME 2003 design engineering technical conference and computers and information in engineering conference (DETC2003), Chicago, Illinois, USA, 2005.

[14] Du,X.P., "Interval reliability analysis", inASME 2007 design engineering technical conference and computers and information in engineering conference (DETC2007), Las Vegas, Nevada, USA, 2007.

[15] Guo, J. and Du, X. P., "Reliability sensitivity analysis with random and interval variables", International journal for numerical methods in engineering, Vol. 78, 2009, pp. 1585-161.

[16] Lu, Z. Z., Feng, Y. W. and Yue, Z. F., "A advanced interval-truncation approach and non-probabilistic reliability analysis based on interval analysis", Chinese Journal of Computational Mechanics, Vol.19, No.3, 2002, pp. 260-264.

[17] Zhao, M. H., Jiang, C. and Cao, W. G., "Nonprobabilistic reliability analysis of retaining walls based on interval theory", Chinese Journal of Geotechnical Engineering, Vol.30, No. 4, 2008, pp. 467-472.

[18] Zou, T. F., Cai, M. and Shu, X., "Response surface methodology and improved interval analysis method-For analyzing uncertainty in accident reconstruction". Forensic Science International222, 2012, pp. 306-312.

[19] Jiang, T., Chen, J. J. and Xu, Y. L.,'A semi-analytic method for calculating non-probabilistic reliability index 
based on interval models". Applied Mathematical Modelling, Vo. 31, 2007, pp. 1362-1370.

[20] Koduru, S. D. and Haukaas, T., "Feasibility of FORM in the finite element reliability analysis". Structural Safety, Vol. 32, No. 2, 2010, pp. 145-153.

[21] Eldred, M. S., Swiler, L. P. and Tang, G., "Mixed aleatory-epistemic uncertainty quantification with stochastic expansions and optimization-based interval estimation" Reliability engineering and system safety, Vol.96, 2011, pp. 1092-1113.

[22] Harsheel R. Shah, Serhat Hosder, and Tyler Winter, "A mixed uncertainty quantification approach with evidence theory and stochastic expansions". $16^{\text {th }}$ AIAA Nondeterministic Approaches conference, AIAA page2014-0298, national harbor, Maryland.

[23] Angel Urbina, Sankaran Mahadevan, and Thomas L. Paez., "Quatification of margins and uncertainties of complex systems in the presence of aleatoric and epstemic uncertainty". Reliability engineering and system safety, Vol. 96, 2011, pp. 1114-1125.

[24] Zhao, Y. G., and Ono, T., "Moment methods for structural reliability". Structural Safety, Vol. 23, No. 1, 2001, pp. $47-75$.

[25] Ren, Y. and Bai, G. C., "New Neural Network Response Surface Methods for Reliability Analysis". Chinese Journal of Aeronautics, Vol. 24, 2011, pp. 25-31.

[26]Yan, P. F. and Zhang, C. S., Artificial neural networks and evolutionary computing 2nd ed., Tsinghua University Press, Beijing, China, 2005.

[27] Herbert, M. G. and Armando, M. A., "Comparison of response surface and neural network with other methods for structural reliability analysis". Structural Safety, Vol. 26, 2004, pp. 49-67.

[28] Deng, J., Gu, D. S., Li, X. B. and Yue, Z. Q., “Structural reliability analysis for implicit performance functions using artificial neural network". Structural Safety, Vol. 27, 2005, pp. 25-48.

[29] Bai, Y. C., Han, X., Jiang, C. and Bi, R.G., "A responsesurface-based structural reliability analysis method by using non-probability convex model", Applied Mathematical Modelling, Vol. 38, 2014, pp. 3834-3847

[30] Gong, Q., Zhang, J.G., TC. L. and W, C. C., "Neural Networks Combined with Importance Sampling Techniques for Reliability Evaluation of Explosive Initiating Device". Chinese Journal of Aeronautics, Vol. 25, 2012, pp. 208-215.

[31]Han, X., Jiang, C., Liu, L.X., Liu, J. and Long, X. Y., "Response-surface-based structural reliability analysis with random and interval mixed uncertainties", Science China Technological Sciences, Vol. 57, 2014, pp. 1322-1334.

[32] Zhang, Y. and der Kiureghian, A., "Two improved algorithms for reliability analysis", reliability and optimization of structural systems, Proceedings of the Sixth IFIP WG7.5 Working Conferences on Reliability and Optimization of Structural Systems, Assisi, Italy. 